\title{
Direct Synthesis of Sulfonamides and Activated Sulfonate Esters from Sulfonic Acids
}

\author{
Stephen Caddick, Jonathan D. Wilden and Duncan B. Judd
}

Supporting Information 
To a solution of triphenylphosphine oxide $(1.11 \mathrm{~g}, 4.0 \mathrm{mmol})$ in degassed DCM $(20 \mathrm{~mL})$ was added trifluoromethanesulfonic anhydride $(0.35 \mathrm{~mL}, 0.56 \mathrm{~g}, 1.8 \mathrm{mmol})$ and the solution allowed to stir for 15 minutes. The pyridine salt of $p$-toluenesulfonic acid $(508 \mathrm{mg}, 1.8 \mathrm{mmol})$ was added and the solution allowed to stir for 30 minutes. A mixture of triethylamine $(0.5 \mathrm{~mL}, 3.6 \mathrm{mmol})$ and allylamine $(0.5 \mathrm{~mL})$ in DCM $(10 \mathrm{~mL})$ was added dropwise at $0{ }^{\circ} \mathrm{C}$ over a period of 5 minutes. The resulting pale yellow solution was allowed to warm to RT and diluted with DCM $(20 \mathrm{~mL})$. The reaction mixture was washed with $2 \mathrm{M} \mathrm{HCl}(3 \times 30 \mathrm{~mL})$, saturated sodium bicarbonate solution $(3 \mathrm{x}$ $30 \mathrm{~mL})$ and water $(30 \mathrm{~mL})$. The organic fraction was dried $\left(\mathrm{MgSO}_{4}\right)$, filtered, the filtrate concentrated in vacuo and the crude residue purified by flash column chromatography (20-40\% ether / petroleum ether $40-60{ }^{\circ} \mathrm{C}$ ) to yield the product as a pale yellow solid (586 $\mathrm{mg}, 1.73 \mathrm{mmol}$, $96 \%$ ). Recrystallisation from DCM / hexane gave the product as white crystals. Data in agreement with literature values. ${ }^{1}$

MP $\quad 63-66{ }^{\circ} \mathrm{C}$ (DCM / hexane) Lit. 63-65 (aq. MeOH).

${ }^{1} \mathbf{H}$ NMR $\delta_{\mathrm{H}}\left(300 \mathrm{MHz}, \mathrm{CDCl}_{3}\right) 7.69(2 \mathrm{H}, \mathrm{d}, J=8.3 \mathrm{~Hz}, 2 \mathrm{x} \mathrm{ArH}), 7.23(2 \mathrm{H}, \mathrm{d}, J=8.2 \mathrm{~Hz}, 2 \mathrm{x}$ $\operatorname{ArH}), 5.63\left(1 \mathrm{H}, \mathrm{ddt}, J=17.1,10.2,5.8 \mathrm{~Hz}, \mathrm{CH}_{2} \mathrm{CH}=\mathrm{CH}_{2}\right), 5.08(1 \mathrm{H}$, app. dq, $J=$ $17.1,1.4 \mathrm{~Hz},=\mathrm{CHH}), 4.99(1 \mathrm{H}$, app. dq, $J=10.2,1.3 \mathrm{~Hz},=\mathrm{CHH}), 4.96(1 \mathrm{H}$, obs. $\mathrm{NH}), 3.48$ (2H, app. tt, $\left.J=6.0,1.4 \mathrm{~Hz}, \mathrm{CH}_{2} \mathrm{CH}=\mathrm{CH}_{2}\right), 2.34\left(3 \mathrm{H}, \mathrm{s}, \mathrm{ArCH}_{3}\right)$.

${ }^{13}$ C NMR $\quad \delta_{\mathrm{C}}\left(75 \mathrm{MHz}, \mathrm{CDCl}_{3}\right) 143.9(\mathrm{~s}, \mathbf{C}(\mathrm{Ar})), 137.3(\mathrm{~s}, \mathbf{C}(\mathrm{Ar})), 133.4\left(\mathrm{~d}, \mathbf{C H}=\mathrm{CH}_{2}\right), 130.1$ (d, $2 \times \mathbf{C H}(\mathrm{Ar})), 127.5$ (d, $2 \times \mathbf{C H}(\mathrm{Ar})), 118.0\left(\mathrm{t}, \mathrm{CH}=\mathbf{C H}_{2}\right), 46.1\left(\mathrm{t}, \mathbf{C H}_{2} \mathrm{NH}\right), 21.9$ (q, $\mathrm{ArCH}_{3}$ ).

FTIR $\quad\left(\mathrm{CH}_{2} \mathrm{Cl}_{2}\right.$ Solution, $\left.\mathrm{cm}^{-1}\right) 3287$ br. s, $3056 \mathrm{~s}, 2987 \mathrm{~m}, 1647 \mathrm{w}, 1598 \mathrm{~m}, 1495 \mathrm{w}, 1495$ m, 1422 s, 1328 s, 1265 s, 1161 s, 1094 s.

\section{$\underline{N \text {-Allyl-ethanesulfonamide (10b) }}$}

Prepared as for 10a to give the product as a colourless oil (242 mg, $1.62 \mathrm{mmol}, 90 \%)$. Data consistent with literature values. ${ }^{2}$

${ }^{1} \mathbf{H}$ NMR $\delta_{\mathrm{H}}\left(300 \mathrm{MHz}, \mathrm{CDCl}_{3}\right) 5.79\left(1 \mathrm{H}, \mathrm{ddt}, J=17.1,10.2,5.8 \mathrm{~Hz}, \mathrm{CH}_{2} \mathrm{CH}=\mathrm{CH}_{2}\right), 5.22$ $(1 \mathrm{H}$, app. dq, $J=17.1,1.5 \mathrm{~Hz},=\mathrm{CHH}), 5.11(1 \mathrm{H}$, app. dq, $J=10.3,1.3,=\mathrm{CHH})$, $5.10(1 \mathrm{H}$, obs. $\mathrm{NH}), 3.65\left(2 \mathrm{H}\right.$, app. tt, $\left.J=6.0,1.5 \mathrm{~Hz}, \mathrm{CH}_{2} \mathrm{CH}=\mathrm{CH}_{2}\right), 2.97(2 \mathrm{H}, \mathrm{q}, J$ $\left.=7.4 \mathrm{~Hz}, \mathrm{CH}_{3} \mathrm{CH}_{2} \mathrm{SO}_{2}\right), 1.28\left(3 \mathrm{H}, \mathrm{t}, J=7.4 \mathrm{~Hz}, \mathrm{CH}_{3} \mathrm{CH}_{2} \mathrm{SO}_{2}\right)$.

${ }^{13} \mathbf{C}$ NMR $\quad \delta_{\mathrm{C}}\left(75 \mathrm{MHz}, \mathrm{CDCl}_{3}\right) 134.2\left(\mathrm{~d}, \mathbf{C H}=\mathrm{CH}_{2}\right), 117.7\left(\mathrm{t}, \mathrm{CH}=\mathrm{CH}_{2}\right), 45.9\left(\mathrm{t}, \mathbf{C H}_{2} \mathrm{NH}\right), 8.5$ (q, $\mathrm{CH}_{3}$ ).

FTIR $\left(\mathrm{CH}_{2} \mathrm{Cl}_{2}\right.$ Solution, $\left.\mathrm{cm}^{-1}\right) 3571$ br. m, 3291 br. m, $1642 \mathrm{w}, 1430 \mathrm{~m}, 1317 \mathrm{~s}, 1282 \mathrm{~m}$, $1142 \mathrm{~s}, 1069 \mathrm{~m}$. 
Pyridine-3-sulfonic acid allylamide

Prepared as for 10a to give the product as an off-white solid (289 $\mathrm{mg}, 1.46 \mathrm{mmol}, 81 \%)$.

MP $\quad 102-104{ }^{\circ} \mathrm{C}(\mathrm{DCM} /$ hexane $)$

${ }^{1} \mathbf{H}$ NMR $\delta_{\mathrm{H}}\left(300 \mathrm{MHz}, \mathrm{CDCl}_{3}\right) 9.07(1 \mathrm{H}, \mathrm{d}, J=2.1 \mathrm{~Hz}, \mathrm{ArH}), 8.80(1 \mathrm{H}, \mathrm{dd}, J=4.8,1.4 \mathrm{~Hz}$, $\operatorname{ArH}), 8.17(1 \mathrm{H}$, app. dt, $J=8.1,1.9 \mathrm{~Hz}, \operatorname{ArH}), 7.48(1 \mathrm{H}, \mathrm{dd}, J=8.1,4.9 \mathrm{~Hz}, \operatorname{ArH})$, $5.72\left(1 \mathrm{H}, \mathrm{ddt}, J=17.1,10.2,5.8 \mathrm{~Hz}, \mathrm{CH}_{2} \mathrm{CH}=\mathrm{CH}_{2}\right), 5.39(1 \mathrm{H}$, br. t, $J=6.0 \mathrm{~Hz}$, NH), $5.18(1 \mathrm{H}$, app. dq, $J=17.1,1.4 \mathrm{~Hz}, \mathrm{CH}=\mathrm{CHH}), 5.11(1 \mathrm{H}$, app. dq, $J=10.2$, $1.2 \mathrm{~Hz}, \mathrm{CH}=\mathrm{CHH}$ ), 3.67 (2H, app tt, $J=6.0,1.5 \mathrm{~Hz}, \mathrm{CH}_{2} \mathrm{CH}=\mathrm{CH}_{2}$ ).

${ }^{13}$ C NMR $\delta_{\mathrm{C}}\left(75 \mathrm{MHz}, \mathrm{CDCl}_{3}\right) 153.5(\mathrm{~d}, \mathbf{C H}(\mathrm{Ar})), 148.3(\mathrm{~d}, \mathbf{C H}(\mathrm{Ar})), 137.4(\mathrm{~s}, \mathbf{C}(\mathrm{Ar}))$, 135.3 (d, $\mathbf{C H}=), 133.0$ (d, $\mathbf{C H}(\mathrm{Ar})), 124.2$ (d, $\mathbf{C H}(\mathrm{Ar})), 118.6\left(\mathrm{t},=\mathbf{C H}_{2}\right), 46.1$ (t, $\mathrm{CH}_{2} \mathrm{NH}$ ).

FTIR $\quad\left(\mathrm{CH}_{2} \mathrm{Cl}_{2}\right.$ Solution, $\left.\mathrm{cm}^{-1}\right) 3056 \mathrm{~m}, 2855 \mathrm{~m}, 2715 \mathrm{~m}, 1645 \mathrm{w}, 1583 \mathrm{~m}, 1495 \mathrm{w}, 1417$ m, $1324 \mathrm{~s}, 1265 \mathrm{~s}, 1165 \mathrm{~s}, 1108 \mathrm{~m}$.

HRMS (ES +ve $)[\mathrm{MH}]^{+}, \mathrm{C}_{8} \mathrm{H}_{10} \mathrm{~N}_{2} \mathrm{O}_{2} \mathrm{~S}$ Requires 199.0536. Found 199.0521.

\section{$\underline{N \text {-Allyl methanesulfonamide }}$}

Prepared as for 10a to give the product as a colourless oil (207 mg, $1.53 \mathrm{mmol}, 85 \%)$. Data consistent with literature values. ${ }^{3}$

${ }^{1} \mathbf{H}$ NMR $\delta_{\mathrm{H}}\left(300 \mathrm{MHz}, \mathrm{CDCl}_{3}\right) 5.86\left(1 \mathrm{H}, \mathrm{ddt}, J=17.0,10.2,5.7 \mathrm{~Hz}, \mathrm{CH}_{2} \mathrm{CH}=\mathrm{CH}_{2}\right), 5.26$ (1H, app. dq, $J=17.0,1.5 \mathrm{~Hz}, \mathrm{CH}=\mathrm{CHH}), 5.19(1 \mathrm{H}$, obs., $\mathrm{NH}), 5.15(1 \mathrm{H}$, app. dq, $J$ $=10.2,1.3 \mathrm{~Hz}, \mathrm{CH}=\mathrm{CHH}), 3.70\left(2 \mathrm{H}\right.$, app. tt, $\left.J=5.9,1.5 \mathrm{~Hz}, \mathrm{CH}_{2} \mathrm{NH}\right), 2.92(3 \mathrm{H}, \mathrm{s}$, $\mathrm{CH}_{3} \mathrm{SO}_{2}$ ).

${ }^{13} \mathbf{C}$ NMR $\delta_{\mathrm{C}}\left(75 \mathrm{MHz}, \mathrm{CDCl}_{3}\right) 134.0\left(\mathrm{~d}, \mathbf{C H}=\mathrm{CH}_{2}\right), 118.0\left(\mathrm{t}, \mathrm{CH}=\mathrm{CH}_{2}\right), 45.9\left(\mathrm{t}, \mathbf{C H}_{2} \mathrm{NH}\right)$, $41.1\left(\mathrm{q}, \mathrm{CH}_{3} \mathrm{SO}_{2}\right)$.

FTIR $\left(\mathrm{CH}_{2} \mathrm{Cl}_{2}\right.$ Solution, $\left.\mathrm{cm}^{-1}\right) 3584$ br. m, 3292 br. s, 3019 w, 2934 w, $1646 \mathrm{w}, 1432 \mathrm{~m}$, $1413 \mathrm{~m}, 1314$ vs, 1150 vs, $1066 \mathrm{~m}$.

\section{Thiophene-2-sulfonic acid allylamide}

Prepared as for 10a to give the product as an off-white solid (300 mg, $1.48 \mathrm{mmol}, 82 \%)$. Data consistent with literature values. ${ }^{4}$

MP $\quad 53-55{ }^{\circ} \mathrm{C}(\mathrm{DCM} /$ hexane $)$ Lit. $54-56{ }^{\circ} \mathrm{C}\left(\mathrm{H}_{2} \mathrm{O}\right)$.

${ }^{1} \mathbf{H}$ NMR $\delta_{\mathrm{H}}\left(300 \mathrm{MHz}, \mathrm{CDCl}_{3}\right) 7.65-7.58(2 \mathrm{H}, \mathrm{m}, 2$ x ArH $), 7.10(1 \mathrm{H}, \mathrm{dd}, J=5.0,3.8 \mathrm{~Hz}$, $\operatorname{ArH}), 5.75\left(1 \mathrm{H}\right.$, ddt, $\left.J=17.1,10.3,5.7 \mathrm{~Hz}, \mathrm{CH}_{2} \mathrm{CH}=\mathrm{CH}_{2}\right), 5.20(1 \mathrm{H}$, app. dq, $J=$ $17.1,1.4 \mathrm{~Hz}, \mathrm{CH}=\mathrm{CHH}), 5.12(1 \mathrm{H}$, app. dq, $J=10.3,1.4 \mathrm{~Hz}, \mathrm{CH}=\mathrm{CHH}), 4.91(1 \mathrm{H}$, br. t, $J=5.8 \mathrm{~Hz}, \mathrm{NH}), 3.68\left(2 \mathrm{H}\right.$, app. tt, $\left.J=5.9,1.4 \mathrm{~Hz}, \mathrm{CH}_{2} \mathrm{NH}\right)$.

${ }^{13}$ C NMR $\delta_{\mathrm{C}}\left(75 \mathrm{MHz}, \mathrm{CDCl}_{3}\right) 141.2(\mathrm{~s}, \mathbf{C}(\mathrm{Ar})), 133.0(\mathrm{~d}, \mathbf{C H}(\mathrm{Ar})), 132.7\left(\mathrm{~d}, \mathbf{C H}=\mathrm{CH}_{2}\right)$, 132.4 (d, $\mathbf{C H}(\mathrm{Ar})), 127.9$ (d, $\mathbf{C H}(\mathrm{Ar})), 118.3$ (t, $\left.\mathbf{C H}=\mathbf{C H}_{2}\right), 46.4\left(\mathrm{t}, \mathbf{C H}_{2} \mathrm{NH}\right)$.

FTIR $\left(\mathrm{CH}_{2} \mathrm{Cl}_{2}\right.$ Solution, $\left.\mathrm{cm}^{-1}\right) 3290$ br. s, $3094 \mathrm{~m}, 3058 \mathrm{~m}, 2987 \mathrm{w}, 1646 \mathrm{w}, 1508 \mathrm{w}, 1406$ s, 1336 br. s, 1266 s, 1227 s, 1159 s, 1067 s, 1019 s. 


\section{5-Dimethylamino-naphthalene-1-sulfonic acid allylamide}

Prepared as for 10a to give the product as a green solid (250 $\mathrm{mg}, 0.86 \mathrm{mmol}, 48 \%)$.

\begin{tabular}{|c|c|}
\hline MP & $4-86{ }^{\circ} \mathrm{C}(\mathrm{DCM} / \mathrm{hexane})$ \\
\hline H NMR & $\begin{array}{l}\delta_{\mathrm{H}}\left(300 \mathrm{MHz}, \mathrm{CDCl}_{3}\right) 8.55(1 \mathrm{H}, \mathrm{d}, J=8.5 \mathrm{~Hz}, \mathrm{ArH}), 8.31(1 \mathrm{H}, \mathrm{d}, J=8.7 \mathrm{~Hz}, \mathrm{ArH}), \\
8.26(1 \mathrm{H}, \mathrm{dd}, J=7.3,1.2 \mathrm{~Hz}, \mathrm{ArH}), 7.61-7.48(2 \mathrm{H}, \mathrm{m}, 2 \mathrm{x} \mathrm{ArH}), 7.19(1 \mathrm{H}, \mathrm{d}, J= \\
7.7 \mathrm{~Hz}, \mathrm{ArH}), 5.63\left(1 \mathrm{H}, \mathrm{ddt}, J=17.1,10.2,5.8 \mathrm{~Hz}, \mathrm{CH}_{2} \mathrm{CH}=\mathrm{CH}_{2}\right), 5.09(1 \mathrm{H}, \text { app. } \\
\text { dq, } J=17.1,1.5 \mathrm{~Hz}, \mathrm{CH}=\mathrm{CHH}), 5.00(1 \mathrm{H}, \mathrm{app} . \mathrm{dq}, J=10.2,1.4 \mathrm{~Hz}, \mathrm{CH}=\mathrm{CHH}), \\
4.87(1 \mathrm{H}, \mathrm{br} . \mathrm{t}, J=6.0 \mathrm{~Hz}, \mathrm{NH}), 3.54\left(2 \mathrm{H}, \text { app. tt, } J=6.0,1.4 \mathrm{~Hz}, \mathrm{CH} \mathbf{H}_{2} \mathrm{NH}\right), 2.89 \\
\left(6 \mathrm{H}, \mathrm{s}, \mathrm{N}\left(\mathrm{CH}_{3}\right)_{2}\right) .\end{array}$ \\
\hline C & $\begin{array}{l}\delta_{\mathrm{C}}\left(75 \mathrm{MHz}, \mathrm{CDCl}_{3}\right) 152.4(\mathrm{~s}, \mathbf{C}(\mathrm{Ar})), 135.0(\mathrm{~s}, \mathbf{C}(\mathrm{Ar})), 133.4\left(\mathrm{~d}, \mathbf{C H}=\mathrm{CH}_{2}\right), 131.0 \\
(\mathrm{~d}, \mathbf{C H}(\mathrm{Ar})), 130.3(\mathrm{~s}, \mathbf{C}(\mathrm{Ar})), 130.1(\mathrm{~d}, \mathbf{C H}(\mathrm{Ar})), 130.0(\mathrm{~s}, \mathbf{C}(\mathrm{Ar})), 128.8(\mathrm{~d}, \mathbf{C H} \\
(\mathrm{Ar})), 123.6(\mathrm{~d}, \mathbf{C H}(\mathrm{Ar})), 119.1(\mathrm{~d}, \mathbf{C H}(\mathrm{Ar})), 118.0\left(\mathrm{t}, \mathrm{CH}=\mathbf{C H}_{2}\right), 115.6(\mathrm{~d}, \mathbf{C H} \\
(\mathrm{Ar})), 46.2\left(\mathrm{t}, \mathbf{C H}_{2} \mathrm{NH}\right), 45.8\left(\mathrm{q}, 2 \times \mathbf{C H}_{3}\right) .\end{array}$ \\
\hline & $\begin{array}{l}\left(\mathrm{CH}_{2} \mathrm{Cl}_{2} \text { Solution, } \mathrm{cm}^{-1}\right) 3584 \text { br. w, } 3299 \text { s, } 2943 \text { m, } 2833 \text { m, } 2789 \text { m, } 1646 \text { w, } 1612 \\
\text { w, } 1588 \text { m, } 1574 \text { m, } 1504 \text { w, } 1455 \text { m, } 1409 \text { m, } 1318 \text { s, } 1231 \text { w, } 1161 \text { s, } 1144 \text { s. }\end{array}$ \\
\hline & \\
\hline
\end{tabular}

\section{3-Nitrobenzenesulfonic acid allylamide}

Prepared as for 10a to give the product as a white solid (401 mg, $1.66 \mathrm{mmol}, 92 \%)$. Data consistent with literature values. ${ }^{5}$

MP $\quad 71-74{ }^{\circ} \mathrm{C}(\mathrm{DCM} /$ hexane $)$ Lit.

${ }^{1} \mathbf{H}$ NMR $\quad \delta_{\mathrm{H}}\left(300 \mathrm{MHz}, \mathrm{CDCl}_{3}\right) 8.71(1 \mathrm{H}, \mathrm{t}, J=2.0 \mathrm{~Hz}, \mathrm{ArH}), 8.44(1 \mathrm{H}, \mathrm{ddd}, J=8.2,2.2,1.0$ $\mathrm{Hz}, \operatorname{ArH}), 8.22(1 \mathrm{H}$, br. dt, $J=7.9,1.4 \mathrm{~Hz}, \operatorname{ArH}), 7.76(1 \mathrm{H}, \mathrm{t}, J=8.0 \mathrm{~Hz}, \operatorname{ArH})$, $5.71\left(1 \mathrm{H}\right.$, ddt, $\left.J=17.0,10.2,5.8 \mathrm{~Hz}, \mathrm{CH}_{2} \mathrm{CH}=\mathrm{CH}_{2}\right), 5.18(1 \mathrm{H}$, app. dq, $J=17.1,1.4$ $\mathrm{Hz}, \mathrm{CH}=\mathrm{CHH}), 5.12(1 \mathrm{H}$, app. dq, $J=10.2,1.3 \mathrm{~Hz}, \mathrm{CH}=\mathrm{CHH}), 5.11(1 \mathrm{H}$, obs, $\mathrm{NH}), 3.69$ (2H, app. tt, $\left.J=6.0,1.5 \mathrm{~Hz}, \mathrm{CH}_{2} \mathrm{NH}\right)$.

${ }^{13} \mathbf{C ~ N M R} \delta_{\mathrm{C}}\left(75 \mathrm{MHz}, \mathrm{CDCl}_{3}\right) 148.7(\mathrm{~s}, \mathbf{C}(\mathrm{Ar})), 142.8(\mathrm{~s}, \mathbf{C}(\mathrm{Ar})), 133.1\left(\mathrm{~d}, \mathbf{C H}=\mathrm{CH}_{2}\right), 132.8$ (d, $\mathbf{C H}(\mathrm{Ar})), 131.0$ (d, $\mathbf{C H}(\mathrm{Ar})), 127.6$ (d, CH (Ar)), 122.7 (d, $\mathbf{C H}(\mathrm{Ar})), 118.7$ (t, $\left.\mathrm{CH}=\mathrm{CH}_{2}\right), 46.2\left(\mathrm{t}, \mathbf{C H}_{2} \mathrm{NH}\right)$.

FTIR $\left(\mathrm{CH}_{2} \mathrm{Cl}_{2}\right.$ Solution, $\left.\mathrm{cm}^{-1}\right) 3379$ br. m, $3055 \mathrm{~m}, 2987 \mathrm{~m}, 1646 \mathrm{w}, 1607 \mathrm{w}, 1536 \mathrm{~s}, 1422$ m, 1353 s, 1265 s, 1171 m, 1127 w, 1071 w. 
Prepared as for 10a to give the product as a white solid (360 mg, $1.58 \mathrm{mmol}, 88 \%$ ). Data consistent with literature values. ${ }^{6}$

MP $\quad 46-49{ }^{\circ} \mathrm{C}$ (Ether / hexane) Lit. 45-47 (MeOH).

${ }^{1} \mathbf{H}$ NMR $\quad \delta_{\mathrm{H}}\left(300 \mathrm{MHz}, \mathrm{CDCl}_{3}\right) 7.81(2 \mathrm{H}, \mathrm{d}, J=8.9 \mathrm{~Hz}, 2 \mathrm{x} \mathrm{ArH}), 6.98(2 \mathrm{H}, \mathrm{d}, J=8.9 \mathrm{~Hz}, 2 \mathrm{x}$ $\operatorname{ArH}), 5.71\left(1 \mathrm{H}\right.$, ddt, $\left.J=17.0,10.2,5.8 \mathrm{~Hz}, \mathrm{CH}_{2} \mathrm{CH}=\mathrm{CH}_{2}\right), 5.16(1 \mathrm{H}$, app. dq, $J=$ $\left.17.1,1.4 \mathrm{~Hz}, \mathrm{CH}_{2} \mathrm{CH}=\mathrm{CHH}\right), 5.08\left(1 \mathrm{H}\right.$, app. dq, $\left.J=10.2,1.3 \mathrm{~Hz}, \mathrm{CH}_{2} \mathrm{CH}=\mathrm{CHH}\right)$, $4.75(1 \mathrm{H}$, br. t, $J=6.0 \mathrm{~Hz}, \mathrm{NH}), 3.87\left(3 \mathrm{H}, \mathrm{s}, \mathrm{OCH}_{3}\right), 3.57(2 \mathrm{H}$, br. t, $J=6.0 \mathrm{~Hz}$, $\left.\mathrm{CH}_{2} \mathrm{NH}\right)$.

${ }^{13} \mathbf{C ~ N M R} \quad \delta_{\mathrm{C}}\left(75 \mathrm{MHz}, \mathrm{CDCl}_{3}\right) 163.3(\mathrm{~s}, \mathbf{C}(\mathrm{Ar})), 133.4\left(\mathrm{~d}, \mathbf{C H}=\mathrm{CH}_{2}\right), 131.8(\mathrm{~s}, \mathbf{C}(\mathrm{Ar})), 129.7$ $(\mathrm{d}, 2 \times \mathbf{C H}(\mathrm{Ar})), 118.1$ (t, $\left.\mathrm{CH}=\mathrm{CH}_{2}\right), 114.7\left(\mathrm{~d}, \mathbf{C H}=\mathrm{CH}_{2}\right), 56.0\left(\mathrm{q}, \mathrm{OCH}_{3}\right), 46.1$ (t, $\mathrm{CH}_{2} \mathrm{NH}$ ).

FTIR $\left(\mathrm{CH}_{2} \mathrm{Cl}_{2}\right.$ Solution, $\left.\mathrm{cm}^{-1}\right) 3284$ br. m, $3055 \mathrm{~m}, 2987 \mathrm{w}, 2842 \mathrm{w}, 2305 \mathrm{w}, 1646 \mathrm{w}$, 1598 s, 1579 s, 1498 s, 1421 s, 1329 s, 1264 vs, 1157 vs, 1096 m.

\section{$\underline{\text { Pentafluorophenyl Tosylate (11a) }}$}

To a solution of triphenylphosphine $(1.1 \mathrm{~g}, 4.0 \mathrm{mmol}, 2.1 \mathrm{eq}$. $)$ in freshly distilled DCM $(20 \mathrm{~mL})$ at $0{ }^{\circ} \mathrm{C}$ under nitrogen was added trifluoromethanesulfonic anhydride $(508 \mathrm{mg}, 0.30 \mathrm{~mL}, 1.8 \mathrm{mmol}$, 1.0 eq.) and the resulting clear solution was allowed to stir for 20 minutes. Pyridinium $p$ toluenesulfonate was then added in a single portion and the mixture allowed to stir for 20 minutes or until all of the sulfonic acid salt had dissolved. A pre-mixed solution of pentafluorophenol (368 $\mathrm{mg}, 2.0 \mathrm{mmol}, 1.1 \mathrm{eq})$ and triethylamine $(0.47 \mathrm{~mL}, 2.0 \mathrm{mmol})$ in DCM $(5 \mathrm{~mL})$ was then added via syringe over a period of 10 minutes. The reaction was allowed to warm to RT then diluted with $\operatorname{DCM}($ ca. $30 \mathrm{~mL})$ and washed with $2 \mathrm{M}$ sodium carbonate solution $(3 \times 30 \mathrm{~mL}), 2 \mathrm{M} \mathrm{HCl}(3 \times 30$ $\mathrm{mL})$ water $(2 \times 30 \mathrm{~mL})$, separated, dried $\left(\mathrm{MgSO}_{4}\right)$ and filtered. The filtrate was concentrated in vacuo and the crude product was purified by flash column chromatography ( $20 \%$ ether / petroleum ether $40-60{ }^{\circ} \mathrm{C}$ ) to yield the product as a white solid (584 mg, $\left.1.73 \mathrm{mmol}, 96 \%\right)$. Recrystalisation from DCM-Hexane gave white needles. Data consistent with literature values. ${ }^{7}$

MP $\quad 66-69{ }^{\circ} \mathrm{C}(\mathrm{DCM} / \mathrm{hexane})$. Lit. $64-65^{\circ} \mathrm{C}(\mathrm{EtOH})$.

${ }^{1}$ H NMR $\delta_{\mathrm{H}}\left(300 \mathrm{MHz}, \mathrm{CDCl}_{3}\right) 7.86(2 \mathrm{H}, \mathrm{d}, J=8.3 \mathrm{~Hz}, 2 \mathrm{x} \mathrm{ArH}), 7.41(2 \mathrm{H}, \mathrm{d}, J=8.3 \mathrm{~Hz}, 2 \mathrm{x}$ $\mathrm{ArH}), 2.51\left(3 \mathrm{H}, \mathrm{s}, \mathrm{ArCH}_{3}\right)$.

${ }^{13}$ C NMR $\quad \delta_{\mathrm{C}}\left(75 \mathrm{MHz}, \mathrm{CDCl}_{3}\right) 147.2(\mathrm{~s}, \mathbf{C}(\mathrm{Ar})), 132.1(\mathrm{~s}, \mathbf{C}(\mathrm{Ar})), 130.6(\mathrm{~d}, 2 \times \mathbf{C H}(\mathrm{Ar}))$, 129.0 (d, 2 x $\mathbf{C H}(\mathrm{Ar})), 22.2\left(\mathrm{q}, \mathrm{CH}_{3}\right)$.

FTIR (Thin film, cm ${ }^{-1}$ ) $3055 \mathrm{w}, 2927 \mathrm{~m}, 1653 \mathrm{w}, 1578 \mathrm{w}, 1520 \mathrm{vs}, 1457 \mathrm{~m}, 1410 \mathrm{~m}, 1265$ $\mathrm{s}, 1199 \mathrm{~m}, 1057 \mathrm{~s}$.

\section{Pentafluorophenyl ethanesulfonate (11b)}

Prepared as for 11a to give the product as a colourless oil (428 mg, $1.55 \mathrm{mmol}, 86 \%$ ).

${ }^{1} \mathbf{H}$ NMR $\delta_{\mathrm{H}}\left(300 \mathrm{MHz}, \mathrm{CDCl}_{3}\right) 3.50\left(2 \mathrm{H}, \mathrm{q}, J=7.4 \mathrm{~Hz}, \mathrm{CH}_{2} \mathrm{SO}_{3}\right), 1.63(3 \mathrm{H}, \mathrm{t}, J=7.4 \mathrm{~Hz}$, $\mathrm{CH}_{3} \mathrm{CH}_{2}$

${ }^{13} \mathbf{C ~ N M R} \delta_{\mathrm{C}}\left(75 \mathrm{MHz}, \mathrm{CDCl}_{3}\right) 47.9\left(\mathrm{t}, \mathrm{CH}_{3} \mathrm{CH}_{2} \mathrm{SO}_{3}\right), 8.6\left(\mathrm{q}, \mathrm{CH}_{3} \mathrm{CH}_{2} \mathrm{SO}_{3}\right)$.

FT-IR (Thin film, cm ${ }^{-1}$ ) $2981 \mathrm{~m}, 2872 \mathrm{~m}, 1651 \mathrm{w}, 1520 \mathrm{vs}, 1472 \mathrm{~m}, 1460 \mathrm{~m}, 1318 \mathrm{w}, 1293$ w, 1239 w, 1182 s, 1141 s, 1116 m, 1022 s, 997 vs. 


\section{Pyridine-3-sulfonic acid pentafluorophenyl ester (11c)}

Prepared as for 11a to give the product as an off white solid (474 mg, $1.46 \mathrm{mmol}, 81 \%$ ).

MP $\quad 54-56{ }^{\circ} \mathrm{C}(\mathrm{DCM} /$ hexane $)$.

${ }^{1} \mathbf{H}$ NMR $\quad \delta_{\mathrm{H}}\left(300 \mathrm{MHz}, \mathrm{CDCl}_{3}\right) 9.17(1 \mathrm{H}$, br. d, $J=1.9 \mathrm{~Hz}, \mathrm{ArH}), 8.99(1 \mathrm{H}, \mathrm{dd}, J=4.8,1.5$ $\mathrm{Hz}, \operatorname{ArH}), 8.29(1 \mathrm{H}, \mathrm{ddd}, J=8.1,2.3,1.7 \mathrm{~Hz}, \operatorname{ArH}), 7.60(1 \mathrm{H}, \mathrm{ddd}, J=8.2,4.8,0.9$ $\mathrm{Hz}, \mathrm{ArH})$.

${ }^{13}$ C NMR $\quad \delta_{\mathrm{C}}\left(75 \mathrm{MHz}, \mathrm{CDCl}_{3}\right) 156.0(\mathrm{~d}, \mathbf{C H}(\mathrm{Ar})), 149.4(\mathrm{~d}, \mathbf{C H}(\mathrm{Ar})), 136.5(\mathrm{~d}, \mathbf{C H}(\mathrm{Ar}))$, 132.4 (s, C (Ar)), 124.5 (d, CH (Ar)).

FT-IR $\quad\left(\mathrm{CH}_{2} \mathrm{Cl}_{2}\right.$ solution, $\left.\mathrm{cm}^{-1}\right) 3677 \mathrm{w}, 3063 \mathrm{w}, 1575 \mathrm{~m}, 1520 \mathrm{vs}, 1470 \mathrm{~m}, 1421 \mathrm{~s}, 1399 \mathrm{~s}$, $1329 \mathrm{w}, 1202 \mathrm{~s}, 1107 \mathrm{w}, 1022 \mathrm{~s}, 999 \mathrm{~s}$.

HRMS $\quad(\mathrm{ES}+\mathrm{ve})[\mathrm{MH}]^{+}, \mathrm{C}_{11} \mathrm{H}_{5} \mathrm{O}_{3} \mathrm{NF}_{5} \mathrm{~S}$ Requires 325.9905. Found 325.9914.

\section{Pentafluorophenyl methanesulfonate (11d)}

Prepared as for 11a to give the product as a colourless oil (364 mg, $1.39 \mathrm{mmol}, 77 \%)$. Data consistent with literature values. ${ }^{8}$

${ }^{1} \mathbf{H}$ NMR $\delta_{\mathrm{H}}\left(300 \mathrm{MHz}, \mathrm{CDCl}_{3}\right) 3.50\left(3 \mathrm{H}, \mathrm{s}, \mathrm{CH}_{3} \mathrm{SO}_{3}\right)$.

${ }^{13} \mathbf{C}$ NMR $\quad \delta_{\mathrm{C}}\left(75 \mathrm{MHz}, \mathrm{CDCl}_{3}\right) 47.9\left(\mathrm{q}, \mathrm{CH}_{3} \mathrm{SO}_{3}\right)$.

FT-IR (Thin film, $\mathrm{cm}^{-1}$ ) $2981 \mathrm{~m}, 2872 \mathrm{~m}, 1651 \mathrm{w}, 1520 \mathrm{vs}, 1472 \mathrm{~m}, 1460 \mathrm{~m}, 1318 \mathrm{w}, 1293$ w, 1239 w, 1182 s, 1141 s, 1116 m, 1022 s, 997 vs.

Thiophene-2-sulfonic acid pentafluorophenyl ester (11e)

Prepared as for 11a to give the product as a white solid (452 mg, $1.37 \mathrm{mmol}, 76 \%$ ).

MP $\quad 84-86{ }^{\circ} \mathrm{C}(\mathrm{DCM} /$ hexane $)$

${ }^{1} \mathbf{H}$ NMR $\quad \delta_{\mathrm{H}}\left(300 \mathrm{MHz}, \mathrm{CDCl}_{3}\right) 7.92(1 \mathrm{H}, \mathrm{dd}, J=5.0,1.4 \mathrm{~Hz}, \operatorname{ArH}), 7.86(1 \mathrm{H}, \mathrm{dd}, J=4.0,1.3$ $\mathrm{Hz}, \mathrm{ArH}), 7.28(1 \mathrm{H}, \mathrm{dd}, J=5.0,4.0 \mathrm{~Hz}, \mathrm{ArH})$.

${ }^{13} \mathbf{C ~ N M R} \quad \delta_{\mathrm{C}}\left(75 \mathrm{MHz}, \mathrm{CDCl}_{3}\right) 136.8(\mathrm{~d}, \mathbf{C H}(\mathrm{Ar})), 136.7$ (d, CH (Ar)), 133.9 (s, C (Ar)), 128.5 (d, $\mathbf{C H}(\mathrm{Ar}))$.

FTIR $\left(\mathrm{CH}_{2} \mathrm{Cl}_{2}\right.$ Solution, $\left.\mathrm{cm}^{-1}\right) 3100 \mathrm{w}, 3056 \mathrm{~m}, 2987 \mathrm{~m}, 1651 \mathrm{w}, 1520 \mathrm{vs}, 1470 \mathrm{w}, 1400 \mathrm{~s}$, $1194 \mathrm{~s}, 1096 \mathrm{~m}, 1022 \mathrm{~s}$.

LRMS (EI +ve) $330\left(\mathrm{M}^{+}, 26 \%\right), 147\left(\left[\mathrm{M}-\mathrm{OC}_{6} \mathrm{~F}_{5}\right]^{+}, 100 \%\right)$.

HRMS $\quad(\mathrm{ES}+\mathrm{ve})[\mathrm{M}+\mathrm{Na}]^{+} \mathrm{C}_{10} \mathrm{H}_{3} \mathrm{O}_{3} \mathrm{~S}_{2} \mathrm{~F}_{5} \mathrm{Na}$ Requires 352.9336. Found 352.9325.

\section{5-Dimethylamino-naphthalene-1-sulfonic acid pentafluorophenyl ester (11f)}

Prepared as for 11a to give the product as a green solid (391 mg, $0.94 \mathrm{mmol}, 52 \%$ ).

${ }^{1} \mathbf{H}$ NMR $\delta_{\mathrm{H}}\left(300 \mathrm{MHz}, \mathrm{CDCl}_{3}\right) 8.72(1 \mathrm{H}, \mathrm{d}, J=8.7 \mathrm{~Hz}, \mathrm{ArH}), 8.42(1 \mathrm{H}, \mathrm{d}, J=8.7 \mathrm{~Hz}, \mathrm{ArH})$, $8.21(1 \mathrm{H}, \mathrm{dd}, J=7.3,1.3 \mathrm{~Hz}, \operatorname{ArH}), 7.68(1 \mathrm{H}, \mathrm{dd}, J=8.7,7.7 \mathrm{~Hz}, \operatorname{ArH}), 7.55(1 \mathrm{H}$, $\mathrm{dd}, J=8.5,7.4 \mathrm{~Hz}, \operatorname{ArH}), 7.27(1 \mathrm{H}, \mathrm{d}, J=7.3 \mathrm{~Hz}, \operatorname{ArH}), 2.81\left(6 \mathrm{H}, \mathrm{s}, 2\right.$ x CH $\left.\mathbf{C H}_{3}\right)$.

${ }^{13}$ C NMR $\delta_{\mathrm{C}}\left(75 \mathrm{MHz}, \mathrm{CDCl}_{3}\right) 152.3(\mathrm{~s}, \mathbf{C}(\mathrm{Ar})), 133.5(\mathrm{~d}, \mathbf{C H}(\mathrm{Ar})), 131.3(\mathrm{~d}, \mathbf{C H}(\mathrm{Ar}))$, 131.1 (s, C (Ar)), 130.6 (s, C (Ar)), 130.2 (s, C (Ar)), 129.7 (d, CH (Ar)), 123.3 (d, CH (Ar)), 119.5 (d, CH (Ar)), 116.3 (d, CH (Ar)), 45.8 (q, 2 x $\mathbf{C H}_{3}$ ).

FTIR (Thin film, cm $\left.{ }^{-1}\right) 3433$ br. w, $3056 \mathrm{w}, 2948 \mathrm{~m}, 2793 \mathrm{w}, 1650 \mathrm{w}, 1614 \mathrm{w}, 1587 \mathrm{~m}$, $1571 \mathrm{~m}, 1520$ vs, $1465 \mathrm{~m}, 1412 \mathrm{~m}, 1386 \mathrm{~s}, 1265 \mathrm{~s}, 1205 \mathrm{~m}, 1186 \mathrm{~s}, 1142 \mathrm{~m}$. 


\section{Pentafluorophenyl 3-nitrobenzenesulfonate}

Prepared as for 11a to give the product as a white solid (617 mg, $1.69 \mathrm{mmol}, 94 \%)$.

MP $\quad 64-67{ }^{\circ} \mathrm{C}$ (Ether / hexane)

${ }^{1} \mathbf{H}$ NMR $\quad \delta_{\mathrm{H}}\left(300 \mathrm{MHz}, \mathrm{CDCl}_{3}\right) 8.85(1 \mathrm{H}, \mathrm{t}, J=2.0 \mathrm{~Hz}, \mathrm{ArH}), 8.65(1 \mathrm{H}$, ddd, $J=8.2,2.2,1.0$ $\mathrm{Hz}, \operatorname{ArH}), 8.34(1 \mathrm{H}$, app. dt, $J=8.1,1.4 \mathrm{~Hz}, \operatorname{ArH}), 7.91(1 \mathrm{H}, \mathrm{t}, J=8.1 \mathrm{~Hz}, \operatorname{ArH})$.

${ }^{13} \mathbf{C}$ NMR $\quad \delta_{\mathrm{C}}\left(75 \mathrm{MHz}, \mathrm{CDCl}_{3}\right) 148.8(\mathrm{~s}, \mathbf{C}(\mathrm{Ar})), 137.4(\mathrm{~s}, \mathbf{C}(\mathrm{Ar})), 134.1$ (d, CH (Ar)), 131.5 (d, CH (Ar)), 130.0 (d, CH (Ar)), 124.2 (d, CH (Ar)).

FTIR (Thin film, $\mathrm{cm}^{-1}$ ) $3084 \mathrm{w}, 2882 \mathrm{w}, 1608 \mathrm{w}, 1540 \mathrm{~m}, 1520 \mathrm{vs}, 1470 \mathrm{w}, 1404 \mathrm{~m}, 1353$ s, $1200 \mathrm{~s}, 1022 \mathrm{w}, 1000 \mathrm{vs}$.

LRMS (EI +ve) $369\left(\mathrm{M}^{+}, 59 \%\right), 186\left(\left[\mathrm{M}-\mathrm{C}_{6} \mathrm{~F}_{5}\right]^{+}, 68 \%\right), 76(100 \%)$.

HRMS (ES +ve $)[\mathrm{M}+\mathrm{Na}]^{+} \mathrm{C}_{12} \mathrm{H}_{4} \mathrm{NO}_{5} \mathrm{SF}_{5} \mathrm{Na}$ Requires 391.9628. Found 391.9654.

\section{Pentafluorophenyl 4-methoxybenzenesulfonate}

Prepared as for 11a to give the product as a white solid (471 mg, $1.33 \mathrm{mmol}, 74 \%)$.

MP $\quad 54-56{ }^{\circ} \mathrm{C}($ Ether / hexane $)$

${ }^{1} \mathbf{H}$ NMR $\delta_{\mathrm{H}}\left(300 \mathrm{MHz}, \mathrm{CDCl}_{3}\right) 7.90(2 \mathrm{H}, \mathrm{d}, J=9.0 \mathrm{~Hz}, 2 \mathrm{x} \mathrm{ArH}), 7.06(2 \mathrm{H}, \mathrm{d}, J=9.0 \mathrm{~Hz}, 2 \mathrm{x}$ $\mathrm{ArH}), 3.93\left(3 \mathrm{H}, \mathrm{s}, \mathrm{OCH}_{3}\right)$.

${ }^{13}$ C NMR $\quad \delta_{\mathrm{C}}\left(75 \mathrm{MHz}, \mathrm{CDCl}_{3}\right) 165.4(\mathrm{~s}, \mathbf{C}(\mathrm{Ar})), 131.3(\mathrm{~d}, 2 \times \mathbf{C H}(\mathrm{Ar})), 126.0(\mathrm{~s}, \mathbf{C}(\mathrm{Ar}))$, 115.1 (d, 2 x CH (Ar)), 56.3 (q, $\left.\mathrm{OCH}_{3}\right)$

FTIR $\left(\mathrm{CH}_{2} \mathrm{Cl}_{2}\right.$ Solution, cm $\left.{ }^{-1}\right) 3060 \mathrm{w}, 2949 \mathrm{w}, 2846 \mathrm{w}, 1650 \mathrm{w}, 1596 \mathrm{~s}, 1578 \mathrm{~s}, 1520 \mathrm{~s}$, $1467 \mathrm{~m}, 1392 \mathrm{~s}, 1266 \mathrm{~s}, 1198 \mathrm{~s}, 1172 \mathrm{~s}, 1093 \mathrm{~s}$.

LRMS (EI +ve) $354\left(\mathrm{M}^{+}, 18 \%\right), 171\left(\left[\mathrm{M}-\mathrm{OC}_{6} \mathrm{~F}_{5}\right]^{+}, 100 \%\right)$.

HRMS $\quad(\mathrm{ES}+\mathrm{ve})[\mathrm{M}+\mathrm{Na}]^{+} \mathrm{C}_{13} \mathrm{H}_{7} \mathrm{O}_{4} \mathrm{SF}_{5} \mathrm{Na}$ Requires 376.9877. Found 376.9878

\section{$\underline{\text { 1-(3-Nitro-benzenesulfonyl)-piperidine }}$}

Prepared as for 10a to give the product as an off-white solid (387 mg, $1.51 \mathrm{mmol}, 84 \%)$. Data consistent with literature values. ${ }^{9}$

MP $\quad 107-110{ }^{\circ} \mathrm{C}$ (DCM / hexane). Lit. 108-110 (MeOH).

${ }^{1} \mathbf{H}$ NMR $\delta_{\mathrm{H}}\left(300 \mathrm{MHz}, \mathrm{CDCl}_{3}\right) 8.58(1 \mathrm{H}, \mathrm{t}, J=1.7 \mathrm{~Hz}, \mathrm{ArH}), 8.45(1 \mathrm{H}, \mathrm{ddd}, J=8.2,2.3,1.0$ $\mathrm{Hz}, \operatorname{ArH}), 8.09(1 \mathrm{H}, \mathrm{ddd}, J=7.9,1.7,1.0 \mathrm{~Hz}, \operatorname{ArH}), 7.78(1 \mathrm{H}, \mathrm{dt}, J=8.2,7.8 \mathrm{~Hz}$, $\operatorname{ArH}), 3.05\left(4 \mathrm{H}\right.$, br. $\left.\mathrm{t}, J=5.5 \mathrm{~Hz}, \mathrm{~N}\left(\mathrm{CH}_{2}\right)_{2}\right), 1.73-1.60\left(4 \mathrm{H}, \mathrm{m},\left(\mathrm{CH}_{2}\right)_{2}\right), 1.51-1.40$ $\left(2 \mathrm{H}, \mathrm{m}, \mathrm{CH}_{2}\right)$.

${ }^{13}$ C NMR $\quad \delta_{\mathrm{C}}\left(75 \mathrm{MHz}, \mathrm{CDCl}_{3}\right) 148.7(\mathrm{~s}, \mathbf{C}(\mathrm{Ar})), 139.3(\mathrm{~s}, \mathbf{C}(\mathrm{Ar})), 133.5(\mathrm{~d}, \mathbf{C H}(\mathrm{Ar})), 130.8$ (d, $\mathbf{C H}(\mathrm{Ar})$ ), 127.5 (d, $\mathbf{C H}(\mathrm{Ar})), 123.0$ (d, $\mathbf{C H}(\mathrm{Ar})$ ), 47.3 (t, 2 x $\mathbf{C H}_{2}$ ), 25.5 (t, $2 \mathrm{x}$ $\left.\mathrm{CH}_{2}\right), 23.7\left(\mathrm{t}, \mathrm{CH}_{2}\right)$.

FTIR $\left(\mathrm{CH}_{2} \mathrm{Cl}_{2}\right.$ Solution, $\left.\mathrm{cm}^{-1}\right) 3056 \mathrm{w}, 2948 \mathrm{~m}, 2857 \mathrm{w}, 1780 \mathrm{~m}, 1606 \mathrm{w}, 1536 \mathrm{~s}, 1467 \mathrm{w}$, $1353 \mathrm{~s}, 1178 \mathrm{~s}, 1127 \mathrm{~m}$. 


\section{$\underline{\text { 2-(3-Nitro-benzenesulfonylamino)-benzoic acid ethyl ester }}$}

Prepared as for 10a to give the product as an off-white solid (592 mg, $1.69 \mathrm{mmol}, 94 \%$ ).

MP $\quad 76-78{ }^{\circ} \mathrm{C}\left(\mathrm{CHCl}_{3} /\right.$ hexane $)$

${ }^{1} \mathbf{H}$ NMR $\delta_{\mathrm{H}}\left(300 \mathrm{MHz}, \mathrm{CDCl}_{3}\right) 10.91(1 \mathrm{H}$, br. s, NH), $8.65(1 \mathrm{H}, \mathrm{t}, J=1.9 \mathrm{~Hz}, \mathrm{ArH}), 8.36$ $(1 \mathrm{H}, \mathrm{ddd}, J=8.3,2.3,1.1 \mathrm{~Hz}, \operatorname{ArH}), 8.16(1 \mathrm{H}$, app. dt, $J=8.0,1.3 \mathrm{~Hz}, \operatorname{ArH}), 7.94$ $(1 \mathrm{H}, \mathrm{dd} J=8.0,1.6 \mathrm{~Hz}, \operatorname{ArH}), 7.73-7.63(2 \mathrm{H}, \mathrm{m}, 2 \mathrm{x} \mathrm{ArH}), 7.50$ (1H, app. dt, $J=$ 8.0, 1.5 Hz, ArH), $7.09(1 \mathrm{H}$, br. m, $\mathrm{ArH}), 4.32\left(2 \mathrm{H}, \mathrm{q}, J=7.2 \mathrm{~Hz}, \mathrm{CO}_{2} \mathrm{CH}_{2} \mathrm{CH}_{3}\right)$, $1.35\left(3 \mathrm{H}, \mathrm{t}, J=7.2 \mathrm{~Hz}, \mathrm{CO}_{2} \mathrm{CH}_{2} \mathrm{CH}_{3}\right)$.

${ }^{13}$ C NMR $\quad \delta_{\mathrm{C}}\left(75 \mathrm{MHz}, \mathrm{CDCl}_{3}\right) 168.3(\mathrm{~s}, \mathbf{C}=\mathrm{O}), 148.5(\mathrm{~s}, \mathbf{C}(\mathrm{Ar})), 141.8(\mathrm{~s}, \mathbf{C}(\mathrm{Ar})), 139.9$ (s, C (Ar)), 135.1 (d, CH (Ar)), 133.1 (d, CH (Ar)), 131.8 (d, CH (Ar)), 130.9 (d, CH (Ar)), 127.9 (d, $\mathbf{C H}(\mathrm{Ar})), 124.3$ (d, $\mathbf{C H}(\mathrm{Ar})), 122.8$ (d, $\mathbf{C H}(\mathrm{Ar})), 119.8$ (d, $\mathbf{C H}$ (Ar)), 117.0 (s, $\mathbf{C}(\mathrm{Ar})$ ), 62.4 (t, $\left.\mathrm{CO}_{2} \mathrm{CH}_{2} \mathrm{CH}_{3}\right), 14.5$ (q, $\mathrm{CO}_{2} \mathrm{CH}_{2} \mathrm{CH}_{3}$ ).

FT-IR $\quad\left(\mathrm{CH}_{2} \mathrm{Cl}_{2}\right.$ solution, $\left.\mathrm{cm}^{-1}\right) 3151$ br. w, $3090 \mathrm{w}, 2985 \mathrm{w}, 1685 \mathrm{~s}, 1605 \mathrm{~m}, 1585 \mathrm{~m}, 1537$ s, $1492 \mathrm{~s}, 1410 \mathrm{~m}, 1353 \mathrm{~s}, 1310 \mathrm{~m}, 1255 \mathrm{~s}, 1213 \mathrm{w}, 1178 \mathrm{~s}, 1087 \mathrm{~s}$.

HRMS (ES+) $[\mathrm{M}+\mathrm{Na}]^{+}$Requires 373.0465. Found 373.0459.

\section{$N$-(4-Methyl-benzyl)-3-nitro-benzenesulfonamide}

Prepared as for 10a to give the product as a white solid (511 mg, $1.67 \mathrm{mmol}, 93 \%)$.

MP $\quad 125-127{ }^{\circ} \mathrm{C}\left(\mathrm{CHCl}_{3} /\right.$ hexane $)$

${ }^{1} \mathbf{H}$ NMR $\quad \delta_{\mathrm{H}}\left(300 \mathrm{MHz}, \mathrm{CDCl}_{3}\right) 8.57(1 \mathrm{H}, \mathrm{t}, J=1.9 \mathrm{~Hz}, \mathrm{ArH}), 8.38(1 \mathrm{H}, \mathrm{ddd}, J=8.3,2.2,1.0$ $\mathrm{Hz}, \operatorname{ArH}), 8.13(1 \mathrm{H}$, br. dt, $J=8.1,1.4 \mathrm{~Hz}, \operatorname{ArH}), 7.68(1 \mathrm{H}, \mathrm{t}, J=8.0 \mathrm{~Hz}, \operatorname{ArH})$, $7.04(4 \mathrm{H}$, app. s, 4 x ArH), $5.09(1 \mathrm{H}, \mathrm{t}, J=6.1 \mathrm{~Hz}, \mathrm{NH}), 4.21(2 \mathrm{H}, \mathrm{d}, J=6.0 \mathrm{~Hz}$, $\left.\mathrm{CH}_{2} \mathrm{NH}\right), 2.28\left(3 \mathrm{H}, \mathrm{s}, \mathrm{ArCH}_{3}\right)$.

${ }^{13}$ C NMR $\quad \delta_{\mathrm{C}}\left(75 \mathrm{MHz}, \mathrm{CDCl}_{3}\right) 148.5(\mathrm{~s}, \mathbf{C}(\mathrm{Ar})), 142.9(\mathrm{~s}, \mathbf{C}(\mathrm{Ar})), 138.4(\mathrm{~s}, \mathbf{C}(\mathrm{Ar})), 132.9(\mathrm{~d}$, CH (Ar)), 132.7 (s, C (Ar)), 130.7 (d, CH (Ar)), 129.8 (d, 2 x CH (Ar)), 128.3 (d, 2 x CH (Ar)), 127.3 (d, CH (Ar)), 122.7 (d, $\mathbf{C H}(\mathrm{Ar})$ ), 47.6 (t, $\left.\mathbf{C H}_{2} \mathrm{NH}\right), 21.4$ (q, $\left.\mathrm{ArCH}_{3}\right)$.

FT-IR (Nujol, cm $\left.{ }^{-1}\right) 3246$ s, 2923 s, 1608 m, 1543 m, 1515 w, 1463 s, 1353 s, 1326 s, 1270 $\mathrm{m}, 1159 \mathrm{~s}, 1124 \mathrm{~s}, 1050 \mathrm{~s}$. 


\section{$\underline{(R)-2-(3-N i t r o-b e n z e n e s u l f o n y l a m i n o)-3-p h e n y l-p r o p i o n i c ~ a c i d ~ e t h y l ~ e s t e r ~}$}

Prepared as for 10a to give the product as a white solid (416 mg, $1.10 \mathrm{mmol}, 61 \%)$.

\begin{tabular}{|c|c|}
\hline IP & $-95{ }^{\circ} \mathrm{C}\left(\mathrm{CHCl}_{3} /\right.$ hexane $)$ \\
\hline H NMR & 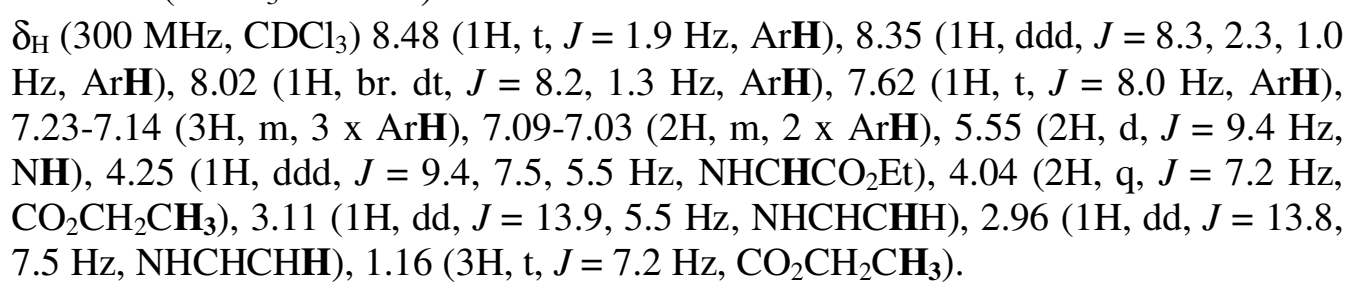 \\
\hline & $\begin{array}{l}\delta_{\mathrm{C}}\left(75 \mathrm{MHz}, \mathrm{CDCl}_{3}\right) 170.9(\mathrm{~s}, \mathbf{C}=\mathrm{O}), 148.2(\mathrm{~s}, \mathbf{C}(\mathrm{Ar})), 142.1(\mathrm{~s}, \mathbf{C}(\mathrm{Ar})), 135.0(\mathrm{~s}, \\
\mathbf{C}(\mathrm{Ar})), 132.6(\mathrm{~d}, \mathbf{C H}(\mathrm{Ar})), 130.4(\mathrm{~d}, \mathbf{C H}(\mathrm{Ar})), 129.4(\mathrm{~d}, 2 \text { x } \mathbf{C H}(\mathrm{Ar})), 128.7(\mathrm{~d}, 2 \\
\text { x } \mathbf{C H}(\mathrm{Ar})), 127.4(\mathrm{~d}, \mathbf{C H}(\mathrm{Ar})), 122.4(\mathrm{~d}, \mathbf{C H}(\mathrm{Ar})), 62.2\left(\mathrm{t}, \mathrm{CO}_{2} \mathbf{C H}_{2} \mathrm{CH}_{3}\right), 57.3(\mathrm{~d}, \\
\mathbf{C H N H}), 39.3\left(\mathrm{t}, \mathrm{NHCHCH}_{2}\right), 14.0\left(\mathrm{q}, \mathrm{CO}_{2} \mathrm{CH}_{2} \mathbf{C H}_{3}\right) .\end{array}$ \\
\hline & $\begin{array}{l}\left(\mathrm{CH}_{2} \mathrm{Cl}_{2} \text { solution, } \mathrm{cm}^{-1}\right) 3340 \mathrm{w}, 3265 \text { br. w, } 3055 \mathrm{~m}, 2986 \mathrm{~m}, 2305 \mathrm{w}, 1734 \mathrm{~s}, 1607 \\
\mathrm{w}, 1536 \mathrm{~s}, 1512 \mathrm{~s}, 1394 \mathrm{~m}, 1358 \mathrm{~s}, 1265 \mathrm{~s}, 1172 \mathrm{~s}, 1127 \mathrm{w}, 1029 \mathrm{w} .\end{array}$ \\
\hline
\end{tabular}

\section{[4-(3-Nitro-benzenesulfonylamino)-phenyl]-acetic acid ethyl ester}

Prepared as for 10a to give the product as a white solid (575 mg, $1.58 \mathrm{mmol}, 88 \%$ ).

\begin{tabular}{|c|c|}
\hline 1 & 9-102 ${ }^{\circ} \mathrm{C}(\mathrm{DCM} /$ hexane $)$ \\
\hline H NMR & ${ }_{\mathrm{H}}\left(300 \mathrm{MHz}, \mathrm{CDCl}_{3}\right) 8.55(1 \mathrm{H}, \mathrm{t}, J=1.9 \mathrm{~Hz}, \mathrm{ArH}), 8.29(1 \mathrm{H}, \mathrm{ddd}, J=8.2,2.1,1.1$ \\
\hline & $\begin{array}{l}\mathrm{Hz}, \mathrm{ArH}), 7.94(1 \mathrm{H}, \text { br. dt, } J=8.1,1.3 \mathrm{~Hz}, \operatorname{ArH}), 7.56(1 \mathrm{H}, \mathrm{t}, J=8.0 \mathrm{~Hz}, \mathrm{ArH}), \\
7.47(1 \mathrm{H}, \mathrm{s}, \mathrm{ArH}), 7.05(2 \mathrm{H}, \mathrm{d}, J=8.5 \mathrm{~Hz}, 2 \mathrm{x} \mathrm{ArH}), 6.88(2 \mathrm{H}, \mathrm{d}, J=8.5 \mathrm{~Hz}, 2 \mathrm{x} \\
\mathrm{ArH}), 4.11\left(2 \mathrm{H}, \mathrm{q}, J=7.1 \mathrm{~Hz}, \mathrm{CO}_{2} \mathrm{CH}_{2} \mathrm{CH}_{3}\right), 3.49\left(2 \mathrm{H}, \mathrm{s}, \operatorname{ArCH}_{2} \mathrm{CO}_{2} \mathrm{Et}\right), 1.19(3 \mathrm{H}, \\
\left.\mathrm{t}, J=7.1 \mathrm{~Hz}, \mathrm{CO}_{2} \mathrm{CH}_{2} \mathrm{CH}_{3}\right) .\end{array}$ \\
\hline $\mathrm{C}$ & $\begin{array}{l}\delta_{\mathrm{C}}\left(75 \mathrm{MHz}, \mathrm{CDCl}_{3}\right) 172.2(\mathrm{~s}, \mathbf{C}=\mathrm{O}), 148.3(\mathrm{~s}, \mathbf{C}(\mathrm{Ar})), 141.2(\mathrm{~s}, \mathbf{C}(\mathrm{Ar})), 134.6(\mathrm{~s}, \\
\mathbf{C}(\mathrm{Ar})), 132.9(\mathrm{~d}, \mathbf{C H}(\mathrm{Ar})), 132.1(\mathrm{~s}, \mathbf{C}(\mathrm{Ar})), 130.6(\mathrm{~d}, 2 \times \mathbf{C H}(\mathrm{Ar})), 130.5(\mathrm{~d}, \mathbf{C H} \\
(\mathrm{Ar})), 127.5(\mathrm{~d}, \mathbf{C H}(\mathrm{Ar})), 122.7(\mathrm{~d}, 2 \times \mathbf{C H}(\mathrm{Ar})), 122.5(\mathrm{~d}, \mathbf{C H}(\mathrm{Ar})), 61.4(\mathrm{t}, \\
\left.\left.\mathrm{CO}_{2} \mathbf{C H}_{2} \mathrm{CH}_{3}\right), 40.6 \mathrm{t}, \mathrm{ArCH}_{2} \mathrm{CO}_{2} \mathrm{Et}\right), 14.2\left(\mathrm{q}, \mathrm{CO}_{2} \mathrm{CH}_{2} \mathbf{C H}_{3}\right) .\end{array}$ \\
\hline IIR & $\begin{array}{l}\left(\mathrm{CH}_{2} \mathrm{Cl}_{2} \text { solution, } \mathrm{cm}^{-1}\right) 3341 \mathrm{w}, 3250 \text { br. w, } 3055 \mathrm{~m}, 2986 \mathrm{~m}, 2305 \mathrm{w}, 1731 \mathrm{~s}, 1607 \\
\mathrm{w}, 1536 \mathrm{~s}, 1512 \mathrm{~m}, 1394 \mathrm{~m}, 1353 \mathrm{~s}, 1265 \mathrm{~s}, 1172 \mathrm{~s}, 1127 \mathrm{w}, 1029 \mathrm{w} .\end{array}$ \\
\hline & \\
\hline
\end{tabular}

\section{$\underline{\text { References }}$}

1. Wedekind, E.; Chem. Ber. 1909, 42, 3939.

2. Whitehead, C. W.; Traverso, J. J.; J. Am. Chem. Soc. 1958, 80, 2182.

3. Tehrani, K. A.; NguyenVan, T.; Karikomi, M.; Rottiers, M.; De Kimpe, N.; Tetrahedron 2002, 58, 7145 .

4. Foye et al. J. Am. Pharm. Assoc. 1952, 41, 273.

5. Patent: Sandoz Ltd., GB 908109, 1962. Chem. Abs. 1963, 58, 11498.

6. Dang. H-S.; Roberts, B. P.; J. Chem. Soc. Perkin Trans. I, 1996, 13, 1493.

7. Forbes et al. J. Chem. Soc. 1959, 2019.

8. Perec, V.; Bae. J-Y.; Zhao, M.; Hill, D. H.; J. Org. Chem. 1995, 60, 176.

9. Bell, C.; J. Chem. Soc. 1933, 1290. 